\title{
Presença de sintomas depressivos em estudantes de medicina de Buenos Aires/Argentina
}

\author{
Presence of depressive symptoms in medical students \\ from Buenos Aires/Argentina
}

\author{
Priscilla Swarça Borsalli ${ }^{1}$, Ana Cláudia Swarça ${ }^{2}$, Solange de Paula Ramos ${ }^{3}$, \\ Raphael Pereira Maia ${ }^{1}$, Elias Jovanovich ${ }^{4}$
}

Resumo

\begin{abstract}
A depressão é um transtorno mental frequente e vários estudos mostram que os estudantes de medicina são mais suscetíveis. O objetivo deste trabalho foi verificar a presença de sintomas depressivos em estudantes de medicina da Universidade Abierta Interamericana, Buenos Aires. Foi utilizada a pesquisa científica descritiva e transversal em que o Inventário de Depressão de Beck foi utilizado. A normalidade dos dados foi avaliada com o teste de Kolmogórov-Smirnov e os dados foram comparados com o teste $\mathrm{T}$ de Student e o teste U de Mann Whitney. As variáveis qualitativas foram comparadas com o teste exato de Fisher. Não houve diferença significativa em idades, níveis de depressão entre homens e mulheres e na distribuição das frequências do ano estudado. O nível leve foi o mais frequente. Os alunos que vivem com amigos têm uma taxa de depressão moderada menor, e os alunos que vivem em hostel têm um índice mais alto. A porcentagem de alunos que nunca fizeram tratamento psicológico é próxima daqueles que nunca receberam tratamento psiquiátrico.
\end{abstract}

Palavras-chave: Distúrbios de adaptação. Estudantes de medicina. Fatores de risco. Argentina.

1 Graduação em Medicina pela Universidad Abierta Interamericana, Facultad de Medicina y Ciências de la Salud, Ciudad Autónoma de Buenos Aires, Província de Buenos Aires, Argentina.

2 Doutorado em Genética pela Universidade Federal do Paraná, Curitiba, Paraná, Brasil. Professora Associada do Departamento de Histologia do Centro de Ciências Biológicas da Universidade Estadual de Londrina, Londrina, Paraná, Brasil. E-mail: swarca@uel.br

3 Doutorado em Medicina e Ciências da Saúde pela Universidade Estadual de Londrina, Londrina, Paraná, Brasil. Professora Associada do Departamento de Histologia do Centro de Ciências Biológicas da Universidade Estadual de Londrina, Londrina, Paraná, Brasil.

4 Graduação em Medicina pela Faculdad de Medicina de la Universidad de Buenos Aires, Ciudad Autónoma de Buenos Aires, Província de Buenos Aires, Argentina. Médico de Staff de Clínica Médica, Hospital Universitário de la Universidad Abierta Interamericana, Facultad de Medicina y Ciências de la Salud, Ciudad Autónoma de Buenos Aires, Província de Buenos Aires, Argentina. 


\begin{abstract}
Depression is a frequent mental disorder and several studies show that medical students are more susceptible. The objective was to verify the presence of depressive symptoms among medical students of the Universidad Abierta Interamericana, Buenos Aires. This is a descriptive and crosssectional scientific study using Beck's Inventory of Depression. Data normality was evaluated using the Kolmogorov-Smirnov test and data were compared with Student's T test and Mann-Whitney $\mathrm{U}$ test. Qualitative variables were compared with the exact test of Fisher. There was no significant difference in age and depression levels between men and women. There were also no differences in the distribution of the absolute and relative frequencies of the year studied. The mild level was the most frequent. Students living with friends had a lower rate of moderate depression, and students living in hostel presented a higher index of this level. The percentage of students who have never had psychological treatment is very close to those who never perform psychiatric treatment.
\end{abstract}

Keywords: Adjustment disorders. Students medical. Risk factors. Argentine.

\section{Introdução}

A depressão é um transtorno do humor e é reconhecida como um problema de saúde pública pela Organização Mundial de Saúde (OMS), considerando sua prevalência em cerca de $15 \%$ da população adulta. Além disso, o subdiagnóstico e o tratamento inadequado de estados depressivos ainda são frequentes. ${ }^{(1)}$ É uma desordem mental que se caracteriza pela presença de tristeza, perda de interesse ou prazer, sentimento de culpa ou falta de autoestima, distúrbios do sono ou do apetite, sensação de cansaço e falta de concentração e afeta mais mulheres do que homens. Na sua forma mais séria pode levar ao suicídio, e de acordo com a OMS no ano 2020, a depressão será a segunda causa da incapacitação no mundo. ${ }^{(2)}$

Nos últimos anos, a saúde mental dos estudantes universitários tornou-se foco de atenção não só de especialistas na área da saúde, mas também da sociedade em geral. $\mathrm{O}$ estudante universitário está constantemente exposto a situações de estresse, como a exigência dos pais, o medo do fracasso ou as imposições do mercado de trabalho. Essas variáveis podem causar quadros de neurose e depressão. ${ }^{(3)}$

Ao analisar a carreira de Medicina, observase que existem inúmeras gratificações inerentes à profissão; entre elas, aliviar o sofrimento, prevenir e curar doenças, diagnosticar corretamente e sentir-se competente, entre outras. ${ }^{(4)}$ Apesar de tudo isso, sabe-se que a carreira médica pode desencadear desequilíbrio emocional com consequências patológicas para a saúde mental ${ }^{(5)}$. Durante o treinamento médico os alunos estão expostos a emoções negativas, como angústia, frustração e medo; ou seja, sensações que podem levar à depressão, ${ }^{(5)}$ além de outros estressores, como a perda de liberdade pessoal, o alto nível de demanda, a sensação de desumanização, a falta de tempo de lazer, a forte competição entre colegas e o contato com pacientes. ${ }^{(6)}$

Conhecendo a gravidade da depressão, vários estudos foram realizados com estudantes de medicina em vários países, como Estados Unidos e Canadá, ${ }^{(7)}$ Turquia, ${ }^{(8)}$ Zimbabwe, ${ }^{(9)}$ Brasil, ${ }^{(10)}$, Peru $^{(11)}$ e Argentina. ${ }^{(12)}$ Todos revelaram uma maior prevalência de sintomas como ansiedade, depressão e desequilíbrios emocionais.

Devido ao interesse na saúde mental do aluno de medicina e ao reconhecimento do desgaste sofrido durante a formação, o objetivo deste estudo foi verificar a prevalência de sintomas depressivos em estudantes de medicina da Universidade Abierta Interamericana, Buenos Aires, avaliando os resultados de acordo com sexo, ano letivo e idade, e de acordo com a convivência do entrevistado. 


\section{Métodos}

Um estudo descritivo e transversal foi realizado com base em uma pesquisa de 150 estudantes de medicina da Universidade Abierta Interamericana (UAI). Os critérios de inclusão do estudo foram: estudantes do primeiro ao sexto ano de medicina, matriculados entre os meses de março e abril do ano de 2015, de ambos os sexos, entre 18 e 36 anos.

Este estudo foi desenvolvido como parte de um trabalho de conclusão de curso e realizado com o consentimento da própria universidade para que o questionário fosse entregue aos alunos. Os alunos concordaram voluntariamente em participar da presente pesquisa, assinando um termo de consentimento livre e esclarecido.

Os dados foram obtidos em dois momentos, um para a aplicação do Inventário de Depressão de Beck (IDB), que permite avaliar a existência de sintomas depressivos e seus graus de gravidade. Em um segundo momento, uma pesquisa para avaliar a associação de sintomas depressivos com sexo, idade, ano de estudo, coexistência e tratamentos psiquiátricos ou psicológicos anteriores da população estudantil.

O questionário consiste em 21 declarações que variam em intensidade de 0 a 3 , com uma pontuação final que pode variar de 0 a 63. Os itens referem-se aos sintomas do caráter afetivo, cognitivo e somático. Os escores obtidos não permitem um diagnóstico definitivo de depressão, mas quantificam o grau de ocorrência de sintomas. É feito através da soma da pontuação de todas as declarações. De 0 a 13 pontos é considerado livre de depressão. De 14 a 19 pontos, ligeiro distúrbio de humor. De 20 a 28 pontos, depressão moderada. De 29 a 63 pontos, depressão grave.

$\mathrm{Na}$ segunda pesquisa foram avaliados os dados dos alunos, sendo analisadas as seguintes variáveis: sexo (Tabela 1), idade (Tabela 2), ano que está estudando (Tabela 3), com quem vive (Tabela 4), se já tiver feito ou tratamento psicológico e se ele já fez ou faz tratamento psiquiátrico.

A normalidade dos dados foi avaliada com o teste de Kolmogorov-Smirnov. Os dados com distribuição normal foram comparados com o teste T de Student, e aqueles com distribuição anormal com o teste U de Mann - Whitney. As variáveis qualitativas foram comparadas com o teste exato de Fisher. As diferenças foram consideradas significativas quando o valor de $\mathrm{P}<0,05$.

\section{Resultados}

No presente estudo foram entrevistadas 82 mulheres $(54,6 \%)$ e 68 homens $(46,4 \%)$, com um total de 150 pessoas. A média de idade dos alunos incluídos na amostra é de 23 anos (25\% a 75\%, quartil: 21-25). O valor mínimo de idade que foi apresentado é de 18 anos, enquanto o valor máximo é de 36 anos. Não houve diferença significativa entre as idades entre homens e mulheres $(\mathrm{P}>0,05$, teste T de Student).

Também não houve diferenças na frequência dos níveis de depressão entre mulheres e homens (Tabela 1, P>0,05, teste exato de Fisher), assim como na distribuição das frequências absoluta e relativa do ano estudado (Tabela $2, \mathrm{P}>0,05$, teste exato de Fisher).

Tabela 1 - Distribuição segundo o sexo e pontuação obtida pelo índice de depressão de Beck (n=150).

\begin{tabular}{rlrrrrrr}
\hline \multicolumn{1}{l}{ Pontuação final } & \multicolumn{2}{c}{ Feminino } & \multicolumn{2}{c}{ Masculino } & \multicolumn{2}{c}{ Total } \\
& & $\mathbf{n}$ & $\mathbf{\%}$ & $\mathbf{n}$ & $\mathbf{\%}$ & \multicolumn{1}{c}{ n } & \% \\
\hline 0-13 & Variação normal do estado de ânimo & 14 & 17,1 & 11 & 16,2 & 25 & 17 \\
13-19 & Depressão leve & 54 & 65,9 & 49 & 72,1 & 103 & 69 \\
$20-28$ & Depressão moderada & 12 & 14,6 & 07 & 10,3 & 19 & 12 \\
29-63 & Depressão grave & 02 & 2,4 & 01 & 1,5 & 03 & 02 \\
\hline
\end{tabular}

Fonte: Autores 
Tabela 2 - Distribuição de frequências absolutas e relativas do ano cursado $(n=150)$.

\begin{tabular}{cc}
\hline Ano cursado & Frequência absoluta (\%) \\
\hline Primeiro & $27(18 \%)$ \\
Segundo & $36(24 \%)$ \\
Terceiro & $21(14 \%)$ \\
Quarto & $31(21 \%)$ \\
Quinto & $19(13 \%)$ \\
Sexto & $16(10 \%)$ \\
\hline
\end{tabular}

Fonte: Autores

A maioria dos indivíduos $(\mathrm{n}=125)$ apresentou depressão severa foram observados em estudantes algum nível de depressão. O nível leve foi o mais de segundo e quarto anos. Observou-se redução frequente com aproximadamente $68 \%(n=103)$. significativa na frequência de depressão no quinto Apesar de uma baixa frequência, os casos de ano (Tabela 3).

Tabela 3 - Distribuição segundo pontuação obtida no índice de depressão de Beck e o ano que está cursando na universidade $(\mathrm{n}=150)$.

\begin{tabular}{ccccccccccc}
\hline $\begin{array}{c}\text { Ano do } \\
\text { curso }\end{array}$ & $\begin{array}{c}\text { Variação normal } \\
\text { do estado de ânimo }\end{array}$ & \multicolumn{2}{c}{ Leve } & \multicolumn{2}{c}{ Moderado } & \multicolumn{2}{c}{ Grave } & \multicolumn{2}{c}{ Total } \\
& $\mathbf{n}$ & $\mathbf{\%}$ & $\mathbf{n}$ & $\mathbf{\%}$ & $\mathbf{n}$ & $\mathbf{\%}$ & $\mathbf{n}$ & $\mathbf{\%}$ & $\mathbf{n}$ & $\mathbf{\%}$ \\
\hline 1 & 03 & 12 & 22 & 21 & 02 & 10,5 & - & - & 27 & 18 \\
2 & 03 & 12 & 27 & 26 & 05 & 26 & 01 & 33 & 36 & 24 \\
3 & 06 & 24 & 12 & 12 & 03 & 16 & - & - & 21 & 14 \\
4 & 02 & 08 & 20 & 19 & 07 & 37 & 02 & 67 & 31 & 21 \\
5 & $07 * *$ & 28 & 12 & 12 & - & - & - & - & 19 & 13 \\
6 & 04 & 16 & 10 & 10 & 02 & 10,5 & - & - & 16 & 10 \\
\hline
\end{tabular}

Fonte: Autores

$(\mathrm{P}>0,05$, teste exato de Fisher).

$* * \mathrm{P}=0,02$.

Os resultados mostraram que, entre os de depressão, independentemente com quem eles acadêmicos, 33\% ( $\mathrm{n}=49)$ viviam sozinhos, $23 \%$ vivem. Os alunos que vivem com amigos tiveram $(n=34)$ com amigos, 13\% $(n=19)$ em casais, 17\% uma menor taxa de depressão moderada do que $(\mathrm{n}=26)$ com pais e $14 \%(\mathrm{n}=22)$ em hostel. A o restante, enquanto para aqueles que vivem em maioria dos indivíduos apresentou algum nível hostel esta variável foi maior (Tabela 4). 
Tabela 4 - Distribuição segundo com quem vive e o grau de depressão segundo o índice de depressão de Beck $(n=150)$.

\begin{tabular}{lccccccccc}
\hline Com quem vive & \multicolumn{2}{c}{$\begin{array}{c}\text { Variação normal } \\
\text { do estado de ânimo }\end{array}$} & \multicolumn{2}{c}{ Leve } & \multicolumn{2}{c}{ Moderado } & \multicolumn{2}{c}{ Grave } & Total \\
& $\mathbf{n}$ & $\mathbf{\%}$ & $\mathbf{n}$ & $\mathbf{\%}$ & $\mathbf{n}$ & $\mathbf{\%}$ & $\mathbf{n}$ & $\mathbf{\%}$ & $\mathbf{n}$ \\
\hline Sozinho & 06 & 24 & 34 & 33 & 08 & 42 & 01 & 33,33 & 49 \\
Com amigos & 09 & 36 & 28 & 27 & $01 *$ & 05 & - & - & 38 \\
Com os pais & 04 & 16 & 26 & 25 & 04 & 21 & 01 & 33,33 & 35 \\
Em casal & 05 & 20 & 11 & 11 & 02 & 11 & 01 & 33,33 & 19 \\
Hostel & 01 & 04 & 04 & 04 & $04 * *$ & 21 & - & - & 09 \\
\hline
\end{tabular}

Fonte: Autores

$(\mathrm{P}>0,05$, teste exato de Fisher).

$* \mathrm{P}=0,04$.

$* * \mathrm{P}=0,01$.

Do número total de estudantes inquiridos, 34 receberam suporte psicológico, quatro estão atualmente sendo tratados e 112 nunca receberam tratamento. Entre estes, nove estão recebendo tratamento psiquiátrico, 22 já o receberam no passado, enquanto 119 nunca procuraram este tipo de tratamento.

\section{Discussão e Conclusão}

No presente trabalho, a presença dos sintomas de depressão em estudantes da carreira médica da UAI foi avaliada comparando essa presença entre os alunos do $1^{\circ}$ ao $6^{\circ}$ ano e os principais fatores associados, como idade, sexo, habitação e tratamentos psicológico e psiquiátrico.

Os alunos foram avaliados separadamente de acordo com o ano em que estão estudando. Este esquema foi proposto por Porcu, Fritzen e Helber, ${ }^{(13)}$ que separaram os alunos em seis amostras independentes, correspondentes a cada ano do curso.

Embora estatisticamente não tenham sido encontradas diferenças entre ambos os sexos, uma população feminina maior pôde ser observada entre os entrevistados, o que mostra uma maior inserção de mulheres na profissão médica. A tendência crescente da participação feminina na medicina tem ocorrido em vários países. ${ }^{(14)}$
A média de idade dos alunos incluídos na amostra é de 23 anos, com um valor mínimo de 18 , enquanto o máximo é de 36 . Não houve diferença significativa entre as idades entre homens e mulheres. Como esperado, a faixa etária dos estudantes universitários tende a seguir o padrão que é mantido em escolas de medicina de forma geral, sendo esta mediana próxima de 22 , como já observado. ${ }^{(15)}$

Ao analisar a frequência dos níveis de depressão entre mulheres e homens, os dados mostraram que não houve diferenças significativas $(\mathrm{P}>0,05)$ entre os dois sexos. Este resultado foi diferente da maioria dos estudos realizados com estudantes de medicina de outras instituições, bem como na população em geral, onde a associação entre sexo feminino e depressão é relatada. ${ }^{(16)}$ Entretanto, alguns resultados coincidem com os dados obtidos neste estudo, onde o sexo não era uma variável estatisticamente significativa. ${ }^{(13,17-19)}$ Avaliando as percentagens do escore obtido no IDB e o ano que estuda na Universidade, observase que a maioria dos indivíduos (83\%) apresenta algum nível de depressão, podendo observar-se uma porcentagem de prevalência de sintomas depressivos leves (68\%) em relação a outros $(17 \%$ nenhum, $12 \%$ moderado, $02 \%$ grave).

Nos primeiros anos, embora os índices não sejam significativos de acordo com os testes 
estatísticos aplicados, os sintomas da depressão estão presentes e isso pode ser devido à entrada na faculdade, o que constitui uma fase de transição em relação ao estilo de vida, de hábitos, diminuição do tempo livre, entre outros, que exige um período de adaptação às diversas mudanças inerentes à vida acadêmica. Podemos afirmar que o volume de informações a serem aprendidas, restrições de tempo, avaliações, competição, questões relativas a relacionamentos íntimos, e aspectos financeiros e familiares foram identificados como agentes estressantes, como já foi relatado por Toews. ${ }^{(20)}$

No que diz respeito ao quarto ano, pode-se dizer que, apesar de ter perdido sua significância estatística, foi o ano em que os quatro níveis de depressão e a maior porcentagem de depressão leve em relação a outros foram manifestados. Aparentemente, este período do curso representa um momento socialmente importante na formação dos alunos, uma vez que ocorre a formação de pequenos grupos em que começam a estudar as disciplinas clínicas, gerando ansiedade e expectativas entre os alunos, sendo este o motivo do aumento dos sintomas leves.

Com relação ao quinto ano, a menor frequência de sintomas depressivos moderados é evidente, provavelmente por ser o momento em que os alunos iniciam assuntos mais relacionados à profissão, e por estarem mais maduros, passaram nos primeiros anos, se adaptaram aos métodos de estudo e à rotina estressante do curso. A expectativa de fazer as disciplinas clínicas acabou por ser um pouco positiva; no entanto, o que não pode ser ignorado é que os alunos que já apresentam uma imagem depressiva e não fazem um tratamento, dificilmente reverterão esses sintomas ou irão desaparecer simplesmente pela supressão dos estressores.

A maioria dos indivíduos que compõem esse estudo têm algum nível de depressão, independentemente de quem compartilhe a vida estudantil, sendo mais frequente a depressão leve, e em nenhuma população predominam as assintomáticas. Estatisticamente, os alunos que vivem com amigos têm uma taxa de depressão moderada menor do que outros. A população avaliada no presente estudo apresenta uma alta taxa de estudantes estrangeiros, sendo observado que a coexistência com os colegas é importante para superar a distância dos membros da família e pode reduzir a prevalência de distúrbios nesse grupo.

Com relação aos resultados obtidos é possível inferir que viver em albergues aumentou a imagem depressiva, uma vez que provavelmente aqueles que optam por esses lugares estão fora de seu habitat e não conhecem ninguém em seu novo destino. Nesse ambiente, o indivíduo compartilha o quarto, coexiste com muitas pessoas diferentes e enfrenta a rotatividade na habitação que, para aqueles que permanecem por muito tempo, pode ficar um tanto desconfortável. Embora possa circular por todo o albergue ou apartamento, o seu quarto, na verdade, é a sua casa. Os quartos são totalmente compartilhados: quarto, banheiro, cozinha, pátio ou varanda. Juntando tudo isso, pode favorecer ou predispor a imagens de ansiedade e, consequentemente, imagens deprimidas. Observamos a relevância desses dados, já que não encontramos na bibliografia que esse tipo de convivência e sua relação em imagens depressivas sejam consideradas.

Do total de alunos entrevistados, 34 (23\%) receberam suporte psicológico, quatro $(2,7 \%)$ estão atualmente sendo tratados e 112 (75\%) nunca receberam tratamento. Esses resultados reforçam os dados encontrados que, apesar de uma grande porcentagem de alunos com depressão leve, consideramos que esses estudantes não buscam ajuda profissional provavelmente como resultado do desconhecimento do seu estado de humor e suas possíveis consequências. O que foi interessante é que os três estudantes que apresentaram depressão grave e um estudante com depressão moderada atualmente estão fazendo um tratamento, provavelmente porque atingiu um nível que requer ajuda profissional. Observamos que, dos entrevistados, consideram apenas o apoio terapêutico os estudantes em quem os sintomas são avançados; seria prudente consultar o profissional nos estágios iniciais e assim evitar chegar em estágios mais sérios. 
Com relação ao tratamento psiquiátrico, nove (6\%) estão recebendo tratamento psiquiátrico, 22 (15\%) já o receberam no passado, enquanto 119 (79\%) nunca foram tratados. Um fato interessante que surge das pesquisas é que a porcentagem de estudantes que nunca fizeram tratamento psicológico é muito próxima daqueles que nunca fizeram tratamento psiquiátrico. Algumas exceções foram detectadas; por exemplo, em alguns casos, alguns estudantes só procuraram ajuda psicológica, outros apenas psiquiátrica. É evidente novamente que os três estudantes que apresentaram depressão grave e os 19 que apresentaram depressão moderada estão atualmente fazendo, ou já fizeram, tratamento psiquiátrico, o que reforça a hipótese de que esses alunos atingiram um nível essencial de ajuda profissional.

É acordado entre vários autores a necessidade de mais pesquisas sobre estudantes de medicina e a adoção de estratégias institucionais para intervir nos quadros psicopatológicos encontrados. ${ }^{(21)}$ Esses aspectos, presentes em várias faculdades de Medicina, podem estar associados ao sofrimento psíquico entre estudantes, o que possibilita a generalização dos resultados e conclusões deste estudo para outras faculdades que apresentam características semelhantes. Portanto, deve ser dada maior atenção à saúde mental desses alunos, que devem ser considerados e avaliados no campo educacional para promover uma melhor adaptação dos alunos. Acreditamos que algumas faculdades de Medicina ainda não consideram o estado emocional dos estudantes e esses distúrbios depressivos podem se tornar crônicos ou mesmo agravar e levar a danos em relação à qualidade do treinamento dos estudantes, futuros médicos.

\section{Conflito de interesses}

Os autores confirmam que não há conflitos de interesses.

\section{Referências}

1 Del Porto JA. Conceito de depressão e seus limites. In: Lafer B, Almeida OP, Fráguas R Jr, Miguel EC, editores. Depressão no ciclo da vida. Porto Alegre: Armad; 2000.

2 Organización Mundial de la Salud. Depresión. 2015 [citado 2015 junio 18]. Disponivel em: http://www.who.int/topics/depression/es/

3 Fortes JRA. Saúde mental do universitário. Neurobiologia 1973; 36:13-24.

4 Cunha MAB, Neves AAF, Moreira ME, Hehn FJ, Lopes TP, Ribeiro CCF, et al. Transtornos psiquiátricos menores e procura por cuidados em estudantes de Medicina. RBEM. 2009;33(3):321-8.

5 Castro FC. Os Temores na Formação e Prática da Medicina: Aspectos Psicológicos. RBEM. 2004;28(1):38-45.

6 Montoya LM, Gutiérrez JA, Toro BE, Briñón MA, Rosas E, Salazar LE. Depresión en estudiantes universitarios y su asociación con el estrés académico. Rev Ces Med. 2010;24(1):7-17.

7 Dyrbye LN, Thomas MR, Shanafelt TD. Systematic review of depression, anxiety and other indicators of psychological distress among U.S. and Canadian medical students. Acad Med. 2006;81(4):354-73.

8 Aktekin M, Karaman T, Senol SE, Erengin H, Akaydin M. Anxiety, depression and stressful life events among medical students: a prospective study in Antalya, Turkey. Med Educ. 2001;35(1):12-7.

9 Vaz RF, Mbajiorgu EF, Acuda SW. A preliminary study of stress levels among first year medical students at the University of Zimbabwe. Cent Afr J Med. 1998;44:214-9.

10 Clark DC, Zillow PB. Vicissitudes of depressed mood during four years of medical school. JAMA. 1988;260(19):2521-8. 
11 Pereyra-Elías R, Ocampo-Mascaró J, SilvaSalazar V, Vélez-Segovia E, Costa-Bullón $\mathrm{AD}$, Toro-Polo LM, et al. Prevalencia y Factores asociados con síntomas depresivos en estudiantes de ciencias de la salud de una universidad privada de Lima, Perú. Rev. Perú. Med. Exp. Salud Publica. 2010;27(4):520-6.

12 Czernik GE, Giménez S, Morel MM, Almirón LM. Variables socio demográficas y síntomas de depresión en estudiantes universitarios de Medicina de Corrientes, Argentina. Alcmeon. 2006;13(2):64-73.

13 Porcu M, Fritzen CV, Helber C. Sintomas depressivos nos estudantes de medicina da Universidade Estadual de Maringá. Psiquiatr. Prat. Med. 2001;34(1):2-6.

14 Ferreira RA, Peret Filho LA, Goulart EMA, Valadão MMA. O estudante de medicina da Universidade Federal de Minas Gerais: perfil e tendências. Rev. Assoc. Med. Bras. 2000;46(3):224-31.

15 Rezende CHA, Abrão CB, Coelho EP, Passos LBS. Prevalência de Sintomas Depressivos entre Estudantes de Medicina da Universidade Federal de Uberlândia. Rev. Bras. Educ. Méd. 2008;32(3):315-23.

16 Mari JJ, Williams PA. A validity study of a psychiatric screening questionnaire (SRQ 20) in primary care in city of São Paulo. Braz. J. Psychiatry. 1986;148:23-6.

17 Facundes VLD, Ludermir AB. Common mental disorders among health care students. Rev. Bras. Psiquiatr. 2005;27:194-200.

18 Lima MC, Domingues MS, Cerqueira AT. Prevalence and risk factors of common mental disorders among medical students. Rev Saúde Públ. 2006;40:1035-41.

19 Almeida AM, Godinho TM, Bittencourt AG, Teles MS, Silva AS, Fonseca DC, Barbosa DBV, Oliveira OS, Costa-Matos E, Rocha CR, Soares AM, Abade B, Oliveira IR. Common mental disorders among medical students. Braz. J. Psychiatry. 2007;56(4):245-51.
20 Toews JA, Lockyer JM, Dobson DJG, Simpson E, Brownell AKW, Brenneis F, MacPherson KM, Cohen GS. Analysis of stress levels among medical students, residents and graduate students at four Canadian schools of medicine. Acad Med. 1997;72(11):997-1002.

21 Souza FGM, Martina MCR, Monteiro FCC, Menezes Neto GC, Ribeiro IB. Anorexia e bulimia nervosa em alunas da faculdade de Medicina da Universidade Federal do Ceará. Rev Psiquiatr Clín. 2002;29(4):172-80. 